\title{
Appropriate Extension of Combined Hepatectomy for Radical Resection of Hilar Cholangiocarcinoma
}

\author{
Shuai Xiang and Xiao-ping Chen*
}

Hepatic Surgery Center, Huazhong University of Science and Technology, China

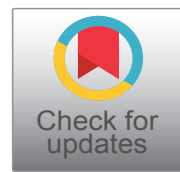

*Corresponding author: Xiao-ping Chen, MD, Ph.D., FACS, Hepatic Surgery Center, Tongji Hospital, Tongji Medical College, Huazhong University of Science and Technology, Wuhan 430030, People's Republic of China, Tel: 0086-27-83663680, Fax: 0086-27-83663500, E-mail: chenxp68599@126.com

\section{Introduction}

Hilar cholangiocarcinoma is a malignant tumor of the bile duct that affects bile duct confluence and its bifurcation. The anatomical location of hilar cholangiocarcinoma is complicated, and the operation is challenging. Most doctors used local bile duct resection for hilar cholangiocarcinoma in the past [1]. Because the biological characteristics of the tumor are growing along the long axis of the bile duct and infiltrating surrounding tissues, surgeons began to recognize that hilar cholangiocarcinoma should be regarded as a regional disease rather than a local lesion as early as in the 1980s. Therefore, combined liver resection is adopted by more and more doctors. In recent years, with the improvement of surgical techniques and medical instruments, morbidity and mortality of surgical resection have decreased significantly. Several retrospective studies from a single center have shown that the proportion of patients undergoing combined hepatectomy progressively increased over time with increased rate of $\mathrm{RO}$ resection and improved survival rate [2-6]. At present, combined liver resection has been widely accepted as a necessary process in the treatment of hilar cholangiocarcinoma. However, how to determine the optimal extent of liver resection remains controversial.

Major liver resection, mainly involving (extensive) hemi-hepatectomy or trisectionectomy, is the method advocated by most surgeons. The primary objective of this resection is to obtain R0 margin as much as possible. There are several characteristics of major liver resection. Firstly, it requires a long time for preoperative preparation. Remnant liver volume after extended hepatectomy is usually inadequate especially in the patients having cholestasis and cholangitis. Therefore, preoperative portal vein embolization should be used to enlarge the future remnant liver volume, and this process usually takes about one month or even longer. Secondly, it results in more surgical trauma. It has been shown that higher postoperative morbidity and mortality were significantly associated with more extended liver resections [5]. The mortality rate after extensive hepatectomy ranged from $4 \%$ up to $25 \%[3,5,7,8]$. Thirdly, there is a higher rate of RO resection. Most surgeons believe that extensive hepatectomy especially trisectionectomy can remove longer bile duct far from the tumor edge, thus increasing the R0 rate [9]. Neuhaus, et al. showed that hilar en bloc resection combined with right trisectionectomy could achieve a 58\% 5-year survival rate [10]. Fourthly, the surgical procedure is relatively simple. After extensive hepatectomy, there is one cross section of the remnant liver, usually 1-3 bile ducts left, and biliary reconstruction is relatively simple.

Minor liver resection mainly refers to the resection of liver segments or subsegments around the hepatic hilum, such as segment $4(4 b), 5$, or segment $4(4 b), 5,8$. The characteristics are as follows, firstly, a large amount of liver parenchyma is preserved, and the morbidity is low. Secondly, the operation is more complex. The resection of the hilar bile duct and the adjacent hepatic parenchyma requires reconstruction of the bile duct openings, which are usually $4-9$ in number, from two

Citation: Xiang S, Xiao-ping C (2018) Appropriate Extension of Combined Hepatectomy for Radical Resection of Hilar Cholangiocarcinoma. Int J Surg Res Pract 5:076. doi.org/10.23937/23783397/1410076

Accepted: May 30, 2018; Published: June 01, 2018

Copyright: (c) 2018 Xiang S, et al. This is an open-access article distributed under the terms of the Creative Commons Attribution License, which permits unrestricted use, distribution, and reproduction in any medium, provided the original author and source are credited. 
transaction planes of the liver. Cholangiojejunostomy for so many bile duct openings are very difficult. As a result of the technical difficulty and worry of inadequate margins, minor liver resection is not favored by most surgeons [11].

Even though resection of the involved intra- and extrahepatic bile ducts as well as the ipsilateral liver for suitable patients is recommended [12], there is no broad consensus on how to determine the appropriate range of hepatectomy for hilar cholangiocarcinoma. It is not advisable to pursue extensive hepatectomy or to adopt minor liver resection without individualized evaluation. Determination of the proper range of hepatectomy needs to solve the following issues.

First of all, anatomy of the bile ducts located in hepatic hilum needs to be well recognized. Some surgeons prefer right-sided hepatectomy in treatment for hilar cholangiocarcinoma. They believe that the left hepatic duct is longer than the right hepatic duct, and that right-sided hepatectomy can resects more bile duct, resulting in a wider tumor free margin. However, a recent study showed that the aforementioned anatomical assumption is a surgeon's biased view. Hirose, et al. conducted studies on the lengths of the hepatic ducts from surgically resected specimens and 3-D images generated from multidetector-row computed tomography (MDCT) and found that the average length of the left hepatic duct and the right hepatic duct were $22.4 \mathrm{~mm}$ and $20.8 \mathrm{~mm}$ respectively, with no statistical difference. When left and right trisectionectomy was performed, the assumed average length of resected right and left bile duct were $31.6 \mathrm{~mm}$ and $34.1 \mathrm{~mm}$ respectively [9]. Even the difference is statistically significant; the length of right bile duct is still comparable with that of the left bile duct.

Secondly, the growth characteristics of hilar cholangiocarcinoma should be well understood. Of all the factors affecting long-term survival of patient with hilar cholangiocarcinoma, RO margin is one of the few factors which can be controlled by a surgeon and the most fundamental goal of the surgical resection for hilar cholangiocarcinoma. Far different from hepatocellular carcinoma which usually forming intrahepatic metastasis via portal veins, hilar cholangiocarcinoma is apt to infiltrate along the bile duct wall. Sakamoto, et al. examined 62 specimens of resected hilar cholangiocarcinoma and found that average invasion distance of mucosal layer from tumor was $11.5 \mathrm{~mm}$, and that was $6 \mathrm{~mm}$ in submucosal layer [13]. The histological data from another larger series of 253 cases reported lengths of $14 \mathrm{~mm}$ and $4.6 \mathrm{~mm}$ in mucosal and submucosal layer respectively. In $79 \%$ of all the cases, the maximum distance of tumor invasion is less than $10 \mathrm{~mm}$ [14]. We also studied 90 specimens from hilar cholangiocarcinoma and found that tumor in filtration along the bile duct mucosa was no longer than $9 \mathrm{~mm}$ in length and into the hepatic pa- renchyma was no longer than $6 \mathrm{~mm}$ in depth. Although it is difficult to determine the exact distance of the tumor cell invasion intraoperatively, the gross margin of $10 \mathrm{~mm}$ seems adequate to guarantee RO margin in most patients. Therefore, there was no sufficient evidence for routinely using major liver resection to obtain RO margins in all patients. Koerkamp, et al. reviewed the recurrence pattern of 306 patients with hilar cholangiocarcinoma after curative intent resection and revealed that the most common sites of tumor recurrence were retroperitoneal lymph nodes, liver, and peritoneum. Only $18 \%$ of the patients, which could have benefited from a more extensive resection or liver transplantation, had isolated local recurrences. Besides, there was no correlation between the disease-free survival rate and the extent of liver resection [15]. Therefore, appropriate extent of liver resection should be determined in the premise of $\mathrm{R} O$ resection.

Thirdly, effective evaluation of tumor invasion is needed both preoperatively and intraoperatively. With the development of medical imaging, it is increasingly likely to assess the invasion extent of the tumor accurately. High resolution MDCT provides thin slice scans, and its speed meets the need for complete examination of the entire upper abdomen during one breath holding. In combination with 3D reconstruction and multiplanar reconstruction (MPR) techniques, MDCT has a high sensitivity for both longitudinal and vertical tumor invasion $[12,16]$. It should be noted that this preoperative imaging examination can only assess the invasion extent of the gross tumor. As mentioned above, the resection site of the bile duct may exceed the gross tumor edge at least $10 \mathrm{~mm}$ to obtain adequate margin. Sasaki underwent preoperative 3D reconstruction of the biliary tract with MDCT, and the image of bile duct stricture was judged to be tumor invasion. They determined the surgical procedure according to the extent of tumor invasion. The average resection margin was $11.4 \mathrm{~mm}$, and the $\mathrm{R} 0$ resection rate was $94 \%$ [17]. Although the sensitivity and specificity of intraoperative frozen section analysis are very limited $[18,19]$, routine frozen section examination is still very important. Studies have shown that when a positive margin is diagnosed, $54 \%-83 \%$ patient can still get RO margin after further resection $[18,20-22]$ and prognosis may improve $[15,20,23]$.

Finally, a convenient and effective cholangiojejunostomy may be established. After extensive hepatectomy, there is only one transection plane of the liver remnant with 1-3 bile ducts requiring reconstruction. The biliary enteric anastomosis is not difficult. However, there are usually 4-9 bile ducts left from two transection planes of the liver remnant after minor liver resection. Procedure of the biliary reconstruction for so many bile ductal openings is quite complex and difficult. We introduced a cholangiojejunostomy which can effectively solve this problem. As described before, resection of liver segment $4 b, 5$ combined with intrahepatic bile ducts are 
performed and the specimen is removed en bloc. The cholangiojejunostomy is made by mucosal to mucosal continuous suturing of the posterior wall of the bile duct to that of the Roux-en-Y loop of jejunum, and the anterior wall of the anastomosis was made between the jejunum and the edge of liver resection adjacent to the biliary ductal openings [24,25]. In animal experiments, we found that the anterior wall of the biliary stoma was everted and sunken in this cholangiojejunostomy and did not result in circular scar, avoiding anastomotic stenosis [24]. Aydin also described a reconstruction technique similar to Kasai procedure, and achieved negative margin without local recurrence for all of the ten patients with type III and IV hilar cholangiocarcinoma [11].

Appropriate extent of liver resection for hilar cholangiocarcinoma should be determined individually. For the tumor only confined to hilar bile duct without vascular invasion and extensive intrahepatic metastasis, minor liver resection is feasible to achieve RO margin in theory, because the tumor is located near the root side of bile duct and the tumor infiltrates no more than 10 $\mathrm{mm}$ along the bile duct wall away from the gross tumor edge in most patients. Large part of the further proximal bile duct and the corresponding liver parenchyma are free of tumor and should be preserved. Most patients with hilar cholangiocarcinoma suffer from obstructive jaundice and cholangitis. Even though major liver resection is technically convenient, the high morbidity and mortality still requires attention.

Determination of the appropriate extent of liver resection for hilar cholangiocarcinoma is not only a technical problem but also an ethical consideration. It is obviously inappropriate to put patients at such high perioperative risks in pursuit of wider margins. Moreover, since minor liver resection can reach the negative margin [11], there is no need to make a trade-off between ensuring radical resection and reducing postoperative risk. In conclusion, with further improvement of diagnostic techniques, preoperative and intraoperative evaluation of tumor invasion extent will be more accurate, and minor liver resection could be successfully performed with RO margin for a considerable number of patients. Extensive liver resection is necessary for patients with vascular invasion or tumor infiltrating the intrahepatic bile duct ending on one side.

\section{References}

1. Boerma EJ (1990) Research into the results of resection of hilar bile duct cancer. Surgery 108: 572-580.

2. Furusawa N, Kobayashi A, Yokoyama T, Shimizu A, Motoyama $\mathrm{H}$, et al. (2014) Surgical treatment of 144 cases of hilar cholangiocarcinoma without liver-related mortality. World J Surg 38: 1164-1176.

3. Nagino M, Ebata T, Yokoyama Y, Igami T, Sugawara G, et al. (2012) Evolution of Surgical Treatment for Perihilar Cholangiocarcinoma: A Single-Center 34-Year Review of 574 Consecutive Resections. Ann Surg 258: 129-140.
4. Kawarada $\mathrm{Y}$, Das BC, Naganuma T, Tabata M, Taoka H (2002) Surgical treatment of hilar bile duct carcinoma: experience with 25 consecutive hepatectomies. J Gastrointest Surg 6: 617-624.

5. Gerhards MF, van Gulik TM, de Wit LT, Obertop H, Gouma DJ (2000) Evaluation of morbidity and mortality after resection for hilar cholangiocarcinoma--a single center experience. Surgery 127: 395-404.

6. Van Gulik TM, Kloek JJ, Ruys AT, Busch OR, van Tienhoven GJ, et al. (2011) Multidisciplinary management of hilar cholangiocarcinoma (Klatskin tumor): extended resection is associated with improved survival. Eur J Surg Oncol 37: 65-71.

7. Tamoto E, Hirano S, Tsuchikawa T, Tanaka E, Miyamoto $M$, et al. (2014) Portal vein resection using the no-touch technique with a hepatectomy for hilar cholangiocarcinoma. HPB (Oxford) 16: 56-61.

8. Molina V, Sampson J, Ferrer J, Diaz A, Ayuso JR, et al. (2017) Surgical treatment of perihilar cholangiocarcinoma: early results of en bloc portal vein resection. Langenbecks Arch Surg 402: 95-104.

9. Hirose T, Igami T, Ebata T, Yokoyama Y, Sugawara G, et al. (2015) Surgical and Radiological Studies on the Length of the Hepatic Ducts. World J Surg 39: 2983-2989.

10. Neuhaus $P$, Thelen A, Jonas S, Puhl G, Denecke T, et al. (2011) Oncological Superiority of Hilar En Bloc Resection for the Treatment of Hilar Cholangiocarcinoma. Ann Surg Oncol 19: 1602-1608.

11. Aydin U, Yedibela S, Yazici P, Aydinli B, Zeytunlu M, et al. (2008) A new technique of biliary reconstruction after "high hilar resection" of hilar cholangiocarcinoma with tumor extension to secondary and tertiary biliary radicals. Ann Surg Oncol 15: 1871-1879.

12. Mansour JC, Aloia TA, Crane $\mathrm{CH}$, Heimbach JK, Nagino $\mathrm{M}$, et al. (2015) Hilar cholangiocarcinoma: expert consensus statement. HPB (Oxford) 17: 691-699.

13. Sakamoto E, Nimura Y, Hayakawa N, Kamiya J, Kondo S, et al. (1998) The pattern of infiltration at the proximal border of hilar bile duct carcinoma: a histologic analysis of 62 resected cases. Ann Surg 227: 405-411.

14. Ebata T, Watanabe H, Ajioka Y, Oda K, Nimura Y (2002) Pathological appraisal of lines of resection for bile duct carcinoma. Br J Surg 89: 1260-1267.

15. Groot Koerkamp B, Wiggers JK, Allen PJ, Besselink MG, Blumgart LH, et al. (2015) Recurrence Rate and Pattern of Perihilar Cholangiocarcinoma after Curative Intent Resection. J Am Coll Surg 221: 1041-1049.

16. Endo I, Matsuyama R, Mori R, Taniguchi K, Kumamoto T, et al. (2014) Imaging and surgical planning for perihilar cholangiocarcinoma. J Hepatobiliary Pancreat Sci 21: 525-532.

17. Sasaki R, Kondo T, Oda T, Murata S, Wakabayashi G, et al. (2011) Impact of three-dimensional analysis of multidetector row computed tomography cholangioportography in operative planning for hilar cholangiocarcinoma. Am J Surg 202: 441-448.

18. Mantel HT, Westerkamp AC, Sieders E, Peeters PM, de Jong KP, et al. (2016) Intraoperative frozen section analysis of the proximal bile ducts in hilar cholangiocarcinoma is of limited value. Cancer Med 5: 1373-1380.

19. Okazaki Y, Horimi T, Kotaka M, Morita S, Takasaki M (2002) Study of the intrahepatic surgical margin of hilar bile duct carcinoma. Hepatogastroenterology 49: 625-627. 
20. Ribero D, Amisano M, Lo Tesoriere R, Rosso S, Ferrero A, et al. (2011) Additional resection of an intraoperative margin-positive proximal bile duct improves survival in patients with hilar cholangiocarcinoma. Ann Surg 254: 776-781.

21. Endo I, House MG, Klimstra DS, Gonen M, D'Angelica M, et al. (2008) Clinical significance of intraoperative bile duct margin assessment for hilar cholangiocarcinoma. Ann Surg Oncol 15: 2104-2112.

22. Lee JH, Hwang DW, Lee SY, Park KM, Lee YJ (2012) The proximal margin of resected hilar cholangiocarcinoma: the effect of microscopic positive margin on long-term survival. Am Surg 78: 471-477.
23. Groot Koerkamp B, Wiggers JK, Gonen M, Doussot A, Allen PJ, et al. (2015) Survival after resection of perihilar cholangiocarcinoma-development and external validation of a prognostic nomogram. Ann Oncol 26: 1930-1935.

24. Yang $H Q$, Xiang $S$, Lau WY, Huang ZY, Luo HP, et al. (2015) A new cholangiojejunostomy for multiple biliary ductal openings: A study in pigs. Int J Surg 22: 15-21.

25. Chen XP, Lau WY, Huang ZY, Zhang ZW, Chen YF, et al. (2009) Extent of liver resection for hilar cholangiocarcinoma. Br J Surg 96: 1167-1175. 